\title{
Perfect Discrimination in Approximate Quantum Theory of General Probabilistic Theories
}

\author{
Yuuya Yoshida $\odot,{ }^{1, *}$ Hayato Arai $\odot,{ }^{1, \dagger}$ and Masahito Hayashi $\oplus^{2,3,4,1, \$}$ \\ ${ }^{1}$ Graduate School of Mathematics, Nagoya University, Furo-cho, Chikusa-ku, Nagoya 464-8602, Japan \\ ${ }^{2}$ Shenzhen Institute for Quantum Science and Engineering, Southern University of Science and Technology, \\ Nanshan District, Shenzhen 518055, China \\ ${ }^{3}$ Guangdong Provincial Key Laboratory of Quantum Science and Engineering, \\ Southern University of Science and Technology, Shenzhen 518055, China \\ ${ }^{4}$ Shenzhen Key Laboratory of Quantum Science and Engineering, Southern University of Science and Technology, \\ Shenzhen 518055, China
}

(Received 13 April 2020; revised 30 July 2020; accepted 10 September 2020; published 9 October 2020)

As a modern approach for the foundation of quantum theory, existing studies of general probabilistic theories gave various models of states and measurements that are quite different from quantum theory. In this Letter, to seek a more realistic situation, we investigate models approximately close to quantum theory. We define larger measurement classes that are smoothly connected with the class of POVMs via a parameter, and investigate the performance of perfect discrimination. As a result, we give a sufficient condition of perfect discrimination, which shows a significant improvement beyond the class of POVMs.

DOI: 10.1103/PhysRevLett.125.150402

Introduction.-Quantum theory (QT) is described by operators on Hilbert spaces, and the description is suitable to represent physical systems. Many researchers have tried to give a foundation of the mathematical description. A modern operational approach that starts with statistics of measurement outcomes is called general probabilistic theories (GPTs) [1-18]. Simply speaking, a GPT is defined by state or measurement classes that satisfy the following postulate. Non-negativity of probability: for each measurement and each state, the probability to obtain each measurement outcome is non-negative.

In QT, the state class and measurement class are given as density matrices and positive-operator valued measures (POVMs), respectively, which indeed satisfy non-negativity of probability. In this way, QT is a typical example of GPTs, and so is classical probability theory (CPT). Unfortunately, there is no operational reason in the sense of GPTs why only QT and CPT describe physical systems. That is, no studies have investigated how one denies an alternative realistic model of GPTs while it is known that there are superior models to QT and CPT with respect to information processing [13-18].

Preceding studies of GPTs defined models by restricting a state class to a much smaller one than QT or CPT. Once restricting a state class, non-negativity of probability

Published by the American Physical Society under the terms of the Creative Commons Attribution 4.0 International license. Further distribution of this work must maintain attribution to the author(s) and the published article's title, journal citation, and DOI. becomes a weaker condition, and the allowed measurement class becomes larger. Consequently, measurement classes of preceding studies are much larger than QT and CPT, and the classes sometimes show superiority of information processing. For example, the PR box, which is defined by restricting states to only convex combinations of four states, violates Bell's inequality more strongly than QT, i.e., exceeds Tsirelson's bound [16,17]. Also, Ref. [18] focuses on the case when available states are restricted to only separable states and all measurements with non-negativity of probability are allowed. The pair of these state and measurement classes is called SEP, and Ref. [18] shows that SEP has the superiority of perfect discrimination of bipartite separable pure states.

However, since the above models are too far from QT, the reality of these models is easily denied. Hence, we should consider measurement classes like $\mathcal{M}_{s}$ in Fig. 1 that are closer to the class of POVMs $\left(\mathcal{M}_{0}\right.$ in Fig. 1$)$ than the measurement class of SEP. If a measurement class is sufficiently close to the class of POVMs, it is hard for an experiment to deny the model because the difference

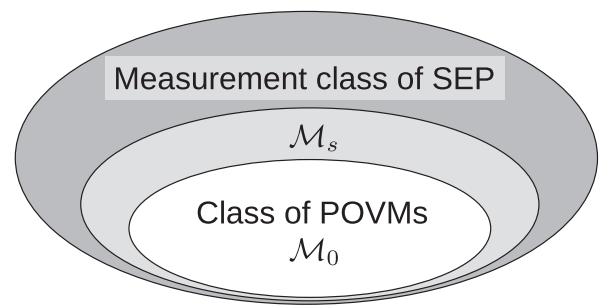

FIG. 1. The measurement classes $\mathcal{M}_{s}$ are smoothly connected with the class $\mathcal{M}_{0}$ of POVMs via the parameter $s$. 
between the experiment and model might be due to an experimental error. In this Letter, in order to deny such an alternative measurement class theoretically, we investigate whether an extended measurement class drastically improves perfect discrimination of separable states even when it sufficiently approximates the class of POVMs. For this aim, we define slightly larger measurement classes than that of POVMs to satisfy the following three conditions, and investigate what happens in adopting them. (i) The measurement classes are larger than the class of POVMs. (ii) Non-negativity of probability holds for every separable state. (iii) The measurement classes are represented as a continuous one-parameter family with the parameter $s$, and the case $s=0$ is just the class of POVMs. For small $s>0$, the measurement class can be regarded as an approximation of the class of POVMs.

We consider two measurement classes. The first one $\mathcal{M}\left(\mathcal{K}_{s}^{\text {neg }}\right)$ is defined by the positive cone $\mathcal{K}_{s}^{\text {neg }}$, which consists of Hermitian matrices allowed to have restricted negative eigenvalues. The second one $\mathcal{M}\left(\mathcal{K}_{s}^{\mathrm{sc}}\right)$ is defined by the positive cone $\mathcal{K}_{s}^{\mathrm{sc}}$, which is given as the sum of positive semidefinite matrices and positive partial transpose of restricted entangled vectors. As a result, we show that the performance of perfect discrimination is dramatically improved unless $s=0$, which implies that the above approximate classes are unlikely to exist.

Framework of GPTs. - Throughout this Letter, we only consider finite-dimensional systems. As already stated, there are state and measurement classes in a GPT. First, let us describe a state class. To handle both pure and mixed states, the set of all states must be a closed convex set. In GPTs, the set $\mathcal{S}(\mathcal{K}, u)$ of all states is defined by the intersection of an affine hyperplane and a positive cone:

$$
\mathcal{S}(\mathcal{K}, u)=\{x \in \mathcal{K} \mid\langle x, u\rangle=1\},
$$

where $\mathcal{K}$ is a positive cone of a finite-dimensional real Hilbert space $\mathcal{V}$ equipped with an inner product $\langle\cdot, \cdot\rangle$, and the unit effect $u$ is an interior point of the dual cone $\mathcal{K}^{*}$. Here, $\mathcal{K}$ is called a positive cone if $\mathcal{K}$ is a closed convex set satisfying that (i) $\alpha x \in \mathcal{K}$ for all $\alpha \geq 0$ and $x \in \mathcal{K}$, (ii) $\mathcal{K}$ has nonempty interior, and (iii) $\mathcal{K} \cap(-\mathcal{K})=\{0\}$.

Also, for a positive cone $\mathcal{K}$, the dual cone $\mathcal{K}^{*}$ is defined as

$$
\mathcal{K}^{*}=\{y \in \mathcal{V} \mid \forall x \in \mathcal{K},\langle x, y\rangle \geq 0\},
$$

which is also a positive cone.

Next, let us describe a measurement class $\mathcal{M}$ [of a GPT with a state class $\mathcal{S}(\mathcal{K}, u)$ ] by using non-negativity of probability. A measurement is given as a family $\left\{y_{i}\right\}_{i=1}^{n}$, where $\{1,2, \ldots, n\}$ denotes the set of outcomes. If a state $x \in \mathcal{S}(\mathcal{K}, u)$ is measured by a measurement $\left\{y_{i}\right\}_{i=1}^{n}$, then each outcome $i$ is obtained with probability $\left\langle x, y_{i}\right\rangle$. Therefore, we need the following postulate.
Postulate 1: non-negativity of probability. - For each state $x \in \mathcal{S}(\mathcal{K}, u)$ and each measurement $\left\{y_{i}\right\}_{i=1}^{n} \in \mathcal{M}$, the family $\left\{\left\langle x, y_{i}\right\rangle\right\}_{i=1}^{n}$ is a probability distribution, i.e., $\left\langle x, y_{i}\right\rangle \geq 0$ for all outcomes $i$, and $\sum_{j=1}^{n}\left\langle x, y_{j}\right\rangle=1$.

Due to Postulate, each measurement element $y_{i}$ must lie in $\mathcal{K}^{*}$. The largest measurement class with Postulate is given as

$$
\mathcal{M}\left(\mathcal{K}^{*}, u\right)=\left\{\begin{array}{l|l}
\left\{y_{i}\right\}_{i=1}^{n} & \begin{array}{c}
n \in \mathbb{N}, \sum_{j=1}^{n} y_{j}=u, \\
y_{i} \in \mathcal{K}^{*}(\forall i)
\end{array}
\end{array}\right\},
$$

but we do not assume that $\mathcal{M}$ is the largest one.

In addition, a measurement class $\mathcal{M}$ must satisfy closedness under fundamental operations. (i) Closedness under random mixing: $\mathcal{M}$ satisfies that the measurement $\left\{p y_{i}+\right.$ $\left.(1-p) y_{i}^{\prime}\right\}_{i=1}^{n}$ belongs to $\mathcal{M}$ for all two measurements $\left\{y_{i}\right\}_{i=1}^{n},\left\{y_{i}^{\prime}\right\}_{i=1}^{n} \in \mathcal{M}$ and all $p \in(0,1)$. (ii) Closedness under coarse graining: $\mathcal{M}$ satisfies that the measurement $\left\{\sum_{i \in S_{j}} y_{i}\right\}_{j=1}^{m}$ belongs to $\mathcal{M}$ for every measurement $\left\{y_{i}\right\}_{i=1}^{n} \in \mathcal{M}$ and every partition $S_{1} \cup \cdots \cup S_{m}=$ $\{1, \ldots, n\}$ of outcomes. To hold (i) and (ii), we need to assume that $\mathcal{M}$ is a set of all cone valued measures.

Postulate 2: cone valued measures. - A measurement class $\mathcal{M}$ is expressed as $\mathcal{M}\left(\mathcal{K}^{\prime}, u\right)$ with a positive cone $\mathcal{K}^{\prime}$, where

$$
\mathcal{M}\left(\mathcal{K}^{\prime}, u\right):=\left\{\begin{array}{l|c}
\left\{y_{i}\right\}_{i=1}^{n} \mid \begin{array}{c}
n \in \mathbb{N}, \sum_{j=1}^{n} y_{j}=u, \\
y_{i} \in \mathcal{K}^{\prime}(\forall i)
\end{array}
\end{array}\right\} .
$$

The measurement class $\mathcal{M}\left(\mathcal{K}^{\prime}, u\right)$ is closed under random mixing and coarse graining, since $\alpha x+\beta y$ lies in $\mathcal{K}^{\prime}$ for all $x, y \in \mathcal{K}^{\prime}$ and $\alpha, \beta \geq 0$. By assuming Postulates and, we consider only measurement classes $\mathcal{M}\left(\mathcal{K}^{\prime}, u\right)$ with $\mathcal{K}^{\prime} \subset \mathcal{K}^{*}$.

Now, let us describe the state and measurement classes of QT by using the above framework of GPTs. Assume the following: (i) $\mathcal{V}$ is the set $\mathcal{T}(\mathcal{H})$ of all Hermitian matrices on a finite-dimensional complex Hilbert space $\mathcal{H}$, (ii) an inner product on $\mathcal{T}(\mathcal{H})$ is given by $\langle X, Y\rangle=\operatorname{Tr} X Y$, (iii) $\mathcal{K}$ is the set $\mathcal{T}_{+}(\mathcal{H})$ of all positive semidefinite matrices on $\mathcal{H}$, (iv) $u$ is the identity matrix $I$ on $\mathcal{H}$, and (v) $\mathcal{M}=\mathcal{M}\left[\mathcal{T}_{+}(\mathcal{H}), I\right]$.

Then $\mathcal{S}\left[\mathcal{T}_{+}(\mathcal{H}), I\right]$ equals the set of all density matrices on $\mathcal{H}$, and $\mathcal{M}$ equals the class of POVMs. Since these classes are usual ones in QT, it turns out that QT is a typical example of GPTs.

Perfect discrimination.-Let $\left\{x_{i}\right\}_{i=1}^{n}$ be a family of $n$ states in $\mathcal{S}(\mathcal{K}, u)$. We say that $\left\{x_{i}\right\}_{i=1}^{n}$ is perfectly distinguishable if there exists a measurement $\left\{y_{j}\right\}_{j=1}^{n} \in \mathcal{M}$ such that $\left\langle x_{i}, y_{j}\right\rangle=\delta_{i j}$, where $\delta_{i j}$ denotes the Kronecker delta. In this Letter, we address the case $n=2$ mainly.

Approximate QT.-We consider a bipartite system of two finite-dimensional quantum systems $\mathcal{H}_{A}$ (Alice's system) 
and $\mathcal{H}_{B}$ (Bob's system), but the bipartite system is not necessarily QT. More precisely, we assume (i), (ii), and (iv) for $\mathcal{H}=\mathcal{H}_{A} \otimes \mathcal{H}_{B}$, but do not necessarily assume (iii) or (v). Let us consider such a bipartite system in the framework of GPTs. When Alice and Bob prepare quantum states $\rho^{A}$ and $\rho^{B}$ independently, the product states $\rho^{A} \otimes \rho^{B}$ is prepared on the bipartite system. Considering the convexity of a state class, we need the following postulate.

Postulate 3: A state class of the bipartite system has all separable states.

Hereinafter, $\mathcal{T}\left(\mathcal{H}_{A}\right)$ is denoted by $\mathcal{T}(A)$. The notations $\mathcal{T}_{+}(A), \mathcal{T}(B), \mathcal{T}_{+}(B), \mathcal{T}(A B)$, and $\mathcal{T}_{+}(A B)$ are similarly defined. Also, since the unit effect of the bipartite system is always $I$, we denote $\mathcal{M}\left(\mathcal{K}^{\prime}, I\right)$ by $\mathcal{M}\left(\mathcal{K}^{\prime}\right)$ simply. Let $\operatorname{SEP}(A ; B)$ be the set

$$
\left\{\begin{array}{l|l}
\sum_{i=1}^{n} X_{i}^{A} \otimes X_{i}^{B} & \begin{array}{c}
n \in \mathbb{N}, X_{i}^{A} \in \mathcal{T}_{+}(A), \\
X_{i}^{B} \in \mathcal{T}_{+}(B)(\forall i)
\end{array}
\end{array}\right\} .
$$

Reference [18] used the largest measurement class $\mathcal{M}\left[\operatorname{SEP}(A ; B)^{*}\right]$ to discriminate two separable pure states, but their measurement class is too far from the class of POVMs. Therefore, we need to define a measurement class that is sufficiently close to the class of POVMs. Moreover, for some state class with Postulate 3, the measurement class must satisfy Postulates 1 and 2.

To define such measurement classes, let us define oneparameter families $\mathcal{K}_{s}^{\text {neg }}$ and $\mathcal{K}_{s}^{\mathrm{sc}}$ of positive cones.

Definition 1: one-parameter family of positive cones, I.-For $s \geq 0$, we define the positive cone $\mathcal{K}_{s}^{\text {neg }}$ as

$$
\mathcal{K}_{s}^{\text {neg }}=\left\{X \in \operatorname{SEP}(A ; B)^{*} \mid \operatorname{neg}(X) \leq s \operatorname{Tr} X\right\},
$$

where the function neg: $\mathcal{T}(A B) \rightarrow[0, \infty)$ is defined as

$$
\operatorname{neg}(X)=\max _{\lambda \text { eigenvalue }}\{-\lambda, 0\} .
$$

Note that the inequality $\operatorname{neg}(X+Y) \leq \operatorname{neg}(X)+\operatorname{neg}(Y)$ holds for all $X, Y \in \mathcal{T}(A B)$.

Definition 2: one-parameter family of positive cones, II.-For a vector $v \in \mathcal{H}_{A} \otimes \mathcal{H}_{B}$, let $\operatorname{sc}(v)$ be the value

$$
\operatorname{sc}(v)= \begin{cases}\lambda_{1} \lambda_{2} & v \neq 0, \\ 0 & v=0\end{cases}
$$

where $\quad \lambda_{1} \geq \lambda_{2} \geq \cdots \geq \lambda_{d}, \quad d=\min \left\{\operatorname{dim} \mathcal{H}_{A}, \operatorname{dim} \mathcal{H}_{B}\right\}$, denote the Schmidt coefficients of $v /\|v\|$. Then, for $s \geq 0$, we define the positive cones $\mathcal{K}_{s}^{(0)}$ and $\mathcal{K}_{s}^{\text {sc }}$ as

$$
\begin{aligned}
\mathcal{K}_{s}^{(0)} & =\operatorname{conv}\left\{|v\rangle\left\langle v|| v \in \mathcal{H}_{A} \otimes \mathcal{H}_{B}, \operatorname{sc}(v) \leq s\right\},\right. \\
\mathcal{K}_{s}^{\mathrm{sc}} & =\mathcal{T}_{+}(A B)+\Gamma\left(\mathcal{K}_{s}^{(0)}\right),
\end{aligned}
$$

where $\operatorname{conv}(\mathcal{X})$ denotes the convex hull of a set $\mathcal{X} \subset$ $\mathcal{T}(A B)$ and $\Gamma$ denotes the partial transpose on Bob's system; i.e., $\Gamma$ is the linear map defined by the tensor product of identity map and transposition.

The value $\operatorname{sc}(v)$ is closely related to negative eigenvalues of $\Gamma(|v\rangle\langle v|)$ as the relation

$$
\|v\|^{2} \operatorname{sc}(v)=\operatorname{neg}[\Gamma(|v\rangle\langle v|)]
$$

holds for every $v \in \mathcal{H}_{A} \otimes \mathcal{H}_{B}$ due to the following reason. If $v \neq 0$, the set of all eigenvalues of $\Gamma(|v\rangle\langle v|) /\|v\|^{2}$ is $\left\{ \pm \lambda_{i} \lambda_{j}, \lambda_{k}^{2} \mid 1 \leq i<j \leq d, 1 \leq k \leq d\right\}$, where $\lambda_{1} \geq \cdots \geq \lambda_{d}$ denote the Schmidt coefficients of $v /\|v\|$. Hence, the inequality $\operatorname{sc}(v) \leq s$ is a restriction of negative eigenvalues of elements of $\mathcal{K}_{s}^{\mathrm{sc}}$. Since the Schmidt coefficients of a unit vector $v \in \mathcal{H}_{A} \otimes \mathcal{H}_{B}$ represent the amount of entanglement about the pure state $|v\rangle\langle v|$, one can regard the inequality $\operatorname{sc}(v) \leq s$ as a restriction of entanglement on the inside of the partial transpose $\Gamma$.

Once the parameter $s$ increases, the positive cones $\mathcal{K}_{s}^{\text {neg }}$, $\mathcal{K}_{s}^{(0)}$, and $\mathcal{K}_{s}^{\text {sc }}$ become larger. Thus, the following inclusion relations hold:

$$
\begin{aligned}
\mathcal{T}_{+}(A B) & =\mathcal{K}_{0}^{\text {neg }} \subset \mathcal{K}_{s}^{\text {neg }} \subset \operatorname{SEP}(A ; B)^{*}, \\
\operatorname{SEP}(A ; B) & =\mathcal{K}_{0}^{(0)} \subset \mathcal{K}_{s}^{(0)} \subset \mathcal{T}_{+}(A B), \\
\mathcal{T}_{+}(A B) & =\mathcal{K}_{0}^{\mathrm{sc}} \subset \mathcal{K}_{s}^{\mathrm{sc}} \subset \operatorname{SEP}(A ; B)^{*} .
\end{aligned}
$$

Note that the classes $\mathcal{M}\left(\mathcal{K}_{0}^{\text {neg }}\right)$ and $\mathcal{M}\left(\mathcal{K}_{0}^{\text {sc }}\right)$ are the class of POVMs. Since $\mathcal{K}_{s}^{(0)}$ satisfies local unitary invariance, i.e., $\left(U_{A} \otimes U_{B}\right) \mathcal{K}_{s}^{(0)}\left(U_{A} \otimes U_{B}\right)^{\dagger}=\mathcal{K}_{s}^{(0)}$ for all unitary matrices $U_{A}$ and $U_{B}$, no positive cones $\mathcal{K}_{s}^{\text {sc }}$ depend on an orthonormal basis of $\mathcal{H}_{B}$ that defines the partial transpose $\Gamma$.

Perfect discrimination in approximate QT.-Let us consider perfect discrimination of separable pure states by measurements in $\mathcal{M}\left(\mathcal{K}_{s}^{\text {neg }}\right)$ and $\mathcal{M}\left(\mathcal{K}_{s}^{\text {sc }}\right)$. First, for separable pure states that are parametrized, we give concrete measurements in the case $\operatorname{dim} \mathcal{H}_{A}=\operatorname{dim} \mathcal{H}_{B}=2$. Let $\rho_{1}$ and $\rho_{2}$ be the separable pure states given as

$$
\begin{aligned}
& \rho_{1}=\left[\begin{array}{ll}
1 & 0 \\
0 & 0
\end{array}\right] \otimes\left[\begin{array}{ll}
1 & 0 \\
0 & 0
\end{array}\right], \\
& \rho_{2}=\left[\begin{array}{cc}
1-\alpha_{1} & \beta_{1} \\
\beta_{1} & \alpha_{1}
\end{array}\right] \otimes\left[\begin{array}{cc}
1-\alpha_{2} & \beta_{2} \\
\beta_{2} & \alpha_{2}
\end{array}\right],
\end{aligned}
$$

where $\alpha_{1}, \alpha_{2} \in[0,1]$ and $\beta_{i}=\sqrt{\alpha_{i}\left(1-\alpha_{i}\right)}$. If the relation

$$
\left(1-\alpha_{1}\right)\left(1-\alpha_{2}\right) \leq 16 s^{2} \alpha_{1} \alpha_{2}
$$

holds for $s \in[0,1 / 4]$, then $\rho_{1}$ and $\rho_{2}$ are perfectly distinguishable by some measurement $\left\{T_{i}+\Gamma\left(T_{i}\right)\right\}_{i=1,2} \in$ $\mathcal{M}\left(\mathcal{K}_{s}^{\text {neg }}\right)$. The measurement $\left\{T_{i}+\Gamma\left(T_{i}\right)\right\}_{i=1,2} \in \mathcal{M}\left(\mathcal{K}_{s}^{\text {neg }}\right)$ 
is given below except for the trivial cases $\alpha_{1}=1$ and $\alpha_{2}=1$. If $\gamma:=\alpha_{1}+\alpha_{2}>1$, then

$$
\begin{aligned}
2 \gamma T_{1} & =\gamma\left|v_{1}\right\rangle\left\langle v_{1}|+(\gamma-1)| v_{2}\right\rangle\left\langle v_{2}|+(\gamma-1)| v_{3}\right\rangle\left\langle v_{3}\right|, \\
v_{1} & =\left[\begin{array}{l}
1 \\
0
\end{array}\right] \otimes\left[\begin{array}{l}
1 \\
0
\end{array}\right]-\frac{\beta_{1} \beta_{2}}{\alpha_{1} \alpha_{2}}\left[\begin{array}{l}
0 \\
1
\end{array}\right] \otimes\left[\begin{array}{l}
0 \\
1
\end{array}\right], \\
v_{2} & =\left[\begin{array}{c}
1 \\
-\beta_{1} / \alpha_{1}
\end{array}\right] \otimes\left[\begin{array}{l}
0 \\
1
\end{array}\right], \quad v_{3}=\left[\begin{array}{l}
0 \\
1
\end{array}\right] \otimes\left[\begin{array}{c}
1 \\
-\beta_{2} / \alpha_{2}
\end{array}\right], \\
T_{2} & =\left(U_{A} \otimes U_{B}\right) T_{1}\left(U_{A} \otimes U_{B}\right)^{\dagger}, \\
U_{A} & =\frac{1}{\sqrt{\alpha_{1}}}\left[\begin{array}{cc}
\beta_{1} & \alpha_{1} \\
\alpha_{1} & -\beta_{1}
\end{array}\right], \quad U_{B}=\frac{1}{\sqrt{\alpha_{2}}}\left[\begin{array}{cc}
\beta_{2} & \alpha_{2} \\
\alpha_{2} & -\beta_{2}
\end{array}\right] ;
\end{aligned}
$$

if $\gamma=1$, then

$$
T_{1}=\frac{1}{2}\left[\begin{array}{cccc}
1 & 0 & 0 & -1 \\
0 & 0 & 0 & 0 \\
0 & 0 & 0 & 0 \\
-1 & 0 & 0 & 1
\end{array}\right], \quad T_{2}=\frac{1}{2}\left[\begin{array}{cccc}
0 & 0 & 0 & 0 \\
0 & 1 & 1 & 0 \\
0 & 1 & 1 & 0 \\
0 & 0 & 0 & 0
\end{array}\right]
$$

If $\mathcal{M}\left(\mathcal{K}_{s}^{\text {neg }}\right)$ and $s \in[0,1 / 4]$ are replaced with $\mathcal{M}\left(\mathcal{K}_{s}^{\text {sc }}\right)$ and $s \in[0,1 / 2]$, then Eq. (3) is replaced with $\left(1-\alpha_{1}\right)\left(1-\alpha_{2}\right) \leq t \alpha_{1} \alpha_{2}$, where $t \in[0,1]$ is a unique number with $s=\sqrt{t} /(1+t)$.

A simple calculation ensures that the above measurements indeed discriminate the states (2) perfectly. As shown in Supplemental Material [19], the above measurements belong to $\mathcal{M}\left(\mathcal{K}_{s}^{\text {neg }}\right)$ and $\mathcal{M}\left(\mathcal{K}_{s}^{\mathrm{sc}}\right)$.

Next, let us consider the general case $\operatorname{dim} \mathcal{H}_{A}$, $\operatorname{dim} \mathcal{H}_{B} \geq 2$. Let $\rho_{1}=\rho_{1}^{A} \otimes \rho_{1}^{B}$ and $\rho_{2}=\rho_{2}^{A} \otimes \rho_{2}^{B}$ be separable pure states. There exist orthonormal bases of $\mathcal{H}_{A}$ and $\mathcal{H}_{B}$ such that $\rho_{1}$ and $\rho_{2}$ are expressed as (2); i.e., their representation matrices are given by the direct sums of the matrices (2) and the zero matrix. Therefore, the general case is reduced to the case $\operatorname{dim} \mathcal{H}_{A}=\operatorname{dim} \mathcal{H}_{B}=2$, and we obtain the following theorems.

Theorem 1: perfect discrimination with $\mathcal{M}\left(\mathcal{K}_{s}^{\text {neg }}\right)$.- - If $\left(\operatorname{Tr} \rho_{1}^{A} \rho_{2}^{A}, \operatorname{Tr} \rho_{1}^{B} \rho_{2}^{B}\right)$ belongs to the set

$$
\left\{(x, y) \in[0,1]^{2} \mid x y \leq 16 s^{2}(1-x)(1-y)\right\}
$$

for $s \in[0,1 / 4]$, then $\rho_{1}$ and $\rho_{2}$ are perfectly distinguishable by some measurement in $\mathcal{M}\left(\mathcal{K}_{s}^{\text {neg }}\right)$.
Theorem 2: perfect discrimination with $\mathcal{M}\left(\mathcal{K}_{s}^{\mathrm{sc}}\right)$.- If $\left(\operatorname{Tr} \rho_{1}^{A} \rho_{2}^{A}, \operatorname{Tr} \rho_{1}^{B} \rho_{2}^{B}\right)$ belongs to the set

$$
\left\{(x, y) \in[0,1]^{2} \mid x y \leq t(1-x)(1-y)\right\}
$$

for $t \in[0,1]$, then $\rho_{1}$ and $\rho_{2}$ are perfectly distinguishable by some measurement in $\mathcal{M}\left(\mathcal{K}_{s}^{\mathrm{sc}}\right)$ with $s=\sqrt{t} /(1+t)$.

Theorems 1 and 2 show the following drastic improvement of multiple-copy state discrimination. Let $\sigma_{1}$ and $\sigma_{2}$ be distinct pure states on a single quantum system. In QT, the nontrivial $n$-copy states $\sigma_{1}^{\otimes n}$ and $\sigma_{2}^{\otimes n}$ never be perfectly distinguishable, where we say that the $n$-copy states $\sigma_{1}^{\otimes n}$ and $\sigma_{2}^{\otimes n}$ are nontrivial if $\sigma_{1}$ and $\sigma_{2}$ are distinct and nonorthogonal. However, it is known [18] that, for some finite $n$, the nontrivial $n$-copy states $\sigma_{1}^{\otimes n}$ and $\sigma_{2}^{\otimes n}$ are perfectly distinguishable by some measurement in $\mathcal{M}\left[\operatorname{SEP}^{*}(A ; B)\right]$. Surprisingly, the same statement is true for the measurement classes $\mathcal{M}\left(\mathcal{K}_{s}^{\text {neg }}\right)$ and $\mathcal{M}\left(\mathcal{K}_{s}^{\text {sc }}\right)$ that are slightly larger than the class of POVMs. To see this fact, regarding the $2 n$-copy states $\sigma_{1}^{\otimes 2 n}=\sigma_{1}^{\otimes n} \otimes \sigma_{1}^{\otimes n}$ and $\sigma_{2}^{\otimes 2 n}=\sigma_{2}^{\otimes n} \otimes \sigma_{2}^{\otimes n}$ as bipartite separable pure states, we apply Theorem 1 to them. Assume $s \in(0,1 / 4]$. Since

$$
x=y=\operatorname{Tr} \sigma_{1}^{\otimes n} \sigma_{2}^{\otimes n}=\left(\operatorname{Tr} \sigma_{1} \sigma_{2}\right)^{n} \stackrel{n \rightarrow \infty}{\longrightarrow} 0,
$$

a sufficiently large $n$ satisfies the inequality in (4). Thus, for some finite $n$, the $2 n$-copy states $\sigma_{1}^{\otimes 2 n}$ and $\sigma_{2}^{\otimes 2 n}$ are perfectly distinguishable by some measurement in $\mathcal{M}\left(\mathcal{K}_{s}^{\mathrm{neg}}\right)$. Also, since a sufficiently large $n$ satisfies the inequality in (5), the same statement is true for $\mathcal{M}\left(\mathcal{K}_{s}^{\mathrm{sc}}\right)$. We summarize these facts as the following corollary and Table I.

Corollary 1: multiple-copy state discrimination.Assume $s \in(0,1 / 4]$. Then, for some finite $n \in \mathbb{N}$, the $2 n$-copy states $\sigma_{1}^{\otimes 2 n}=\sigma_{1}^{\otimes n} \otimes \sigma_{1}^{\otimes n}$ and $\sigma_{2}^{\otimes 2 n}=\sigma_{2}^{\otimes n} \otimes \sigma_{2}^{\otimes n}$ are perfectly distinguishable by some measurement in $\mathcal{M}\left(\mathcal{K}_{s}^{\text {neg }}\right)$. The same statement is true for $\mathcal{M}\left(\mathcal{K}_{s}^{\mathrm{sc}}\right)$ and $s \in(0,1 / 2]$.

The sets (4) and (5) are illustrated as Fig. 2. Once the parameter $s$ decreases, the sets (4) and (5) become smaller. However, the origin is an interior point of both sets unless $s$ is zero. This fact is important to understand Corollary 1 because the point $\left(\left(\operatorname{Tr} \sigma_{1} \sigma_{2}\right)^{n},\left(\operatorname{Tr} \sigma_{1} \sigma_{2}\right)^{n}\right)$ sufficiently approaches the origin as $n$ becomes large enough unless $\operatorname{Tr} \sigma_{1} \sigma_{2}=1$.

TABLE I. Finite-copy perfect discrimination for each measurement class. Assume $s \in(0,1 / 4]$ here.

\begin{tabular}{ccccc}
\hline \hline Measurement class & $\mathcal{M}\left[\mathcal{T}_{+}(A B)\right]$ & $\mathcal{M}\left(\mathcal{K}_{s}^{\text {neg }}\right)$ & $\mathcal{M}\left(\mathcal{K}_{s}^{\text {sc }}\right)$ & $\mathcal{M}\left[\operatorname{SEP}(A ; B)^{*}\right]$ \\
\hline $\begin{array}{c}\text { Perfect discrimination } \\
\text { of nontrivial } n \text {-copy states }\end{array}$ & Impossible & $\begin{array}{c}\text { Possible } \\
\text { for finite } n\end{array}$ & $\begin{array}{c}\text { Possible } \\
\text { for finite } n\end{array}$ & $\begin{array}{c}\text { Possible } \\
\text { for finite } n\end{array}$ \\
\hline \hline
\end{tabular}




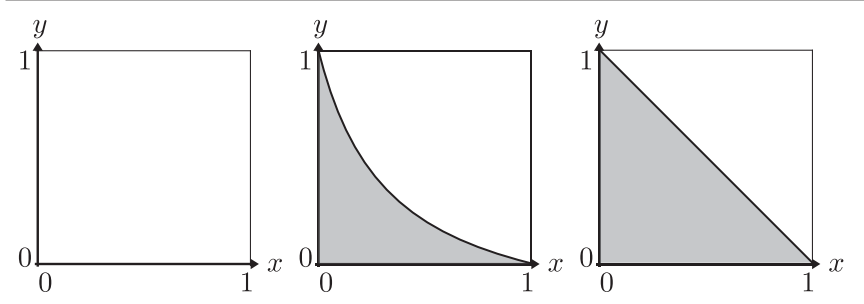

FIG. 2. The sets in Theorem 1 (resp. Theorem 2) for $s=$ $0,1 / 8,1 / 4[\operatorname{resp} .(s, t)=(0,0),(2 / 5,1 / 4),(1 / 2,1)]$.

Conclusion.-To investigate the performance of perfect discrimination in models approximately close to QT, we have defined the two measurement classes $\mathcal{M}\left(\mathcal{K}_{s}^{\text {neg }}\right)$ and $\mathcal{M}\left(\mathcal{K}_{s}^{\mathrm{sc}}\right)$ that are smoothly connected with the class of POVMs $(s=0)$. As a result, unless $s=0$, the performance of perfect discrimination is drastically improved for both $\mathcal{M}\left(\mathcal{K}_{s}^{\text {neg }}\right)$ and $\mathcal{M}\left(\mathcal{K}_{s}^{\mathrm{sc}}\right)$. More precisely, their measurements enable us to discriminate nontrivial $2 n$-copy states perfectly for some finite $n$. This result suggests that the approximate measurement classes $\mathcal{M}\left(\mathcal{K}_{s}^{\text {neg }}\right)$ and $\mathcal{M}\left(\mathcal{K}_{s}^{\text {sc }}\right)$ are unlikely to exist unless $s=0$.

Although we have investigated perfect discrimination, one might be interested in state discrimination that is not necessarily perfect. To investigate such state discrimination, we need to evaluate the minimum error probability in discriminating two separable states for a general measurement class. Unfortunately, this problem is still open even for the measurement classes $\mathcal{M}\left(\mathcal{K}_{s}^{\text {neg }}\right)$ and $\mathcal{M}\left(\mathcal{K}_{s}^{\mathrm{sc}}\right)$.

In this Letter, we have clarified the difference between each approximate measurement class and the class of POVMs. To clarify the difference, one might consider another question when $\mathcal{K}_{s}$ is $\mathcal{K}_{s}^{\text {neg }}$ or $\mathcal{K}_{s}^{\text {sc }}$ : do every measurement in $\mathcal{M}\left(\mathcal{K}_{s}\right)$ except for POVMs have a distinguishable pair of two nonorthogonal states? In this question, strictly speaking, we should consider only measurements $\left\{M_{i}\right\}_{i=1}^{n} \in \mathcal{M}\left(\mathcal{K}_{s}\right)$ such that all $M_{i}$ are on the boundary of $\mathcal{K}_{s}$ because $\operatorname{Tr} X Y>0$ for every nonzero $X \in \mathcal{K}$ and every $Y$ in the interior of $\mathcal{K}_{s}$. The above problem is more difficult mathematically than our results because the question requires us to handle general boundary points. Therefore, the question is another future work.

We have addressed only the possibility of the measurement classes $\mathcal{M}\left(\mathcal{K}_{s}^{\mathrm{neg}}\right)$ and $\mathcal{M}\left(\mathcal{K}_{s}^{\mathrm{sc}}\right)$ as alternative models of QT. However, to deny another possibility beyond these measurement classes, we need to study the following question for a general measurement class $\mathcal{M}$ : is the class $\mathcal{M}$ smaller than the class of POVMs when no measurement in $\mathcal{M}$ discriminates two nonorthogonal $n$-copy states perfectly? If the answer is affirmative, we can deny alternative realistic models in the viewpoint of perfect discrimination. This problem is important for the foundation of QT, but it is a more difficult future work.

To consider the difference between a general measurement class $\mathcal{M}$ and the class of POVMs, we suggest another approach. Assume that there exist states $\sigma_{1}$ and $\sigma_{2}$ such that for every $n \in \mathbb{N}$ the $n$-copy states $\sigma_{1}^{\otimes n}$ and $\sigma_{2}^{\otimes n}$ are not perfectly distinguishable by any measurements in $\mathcal{M}$. Then, focus on the error probability in discriminating $\sigma_{1}^{\otimes n}$ and $\sigma_{2}^{\otimes n}$. If $\mathcal{M}$ is the class of POVMs, the error probability is exponentially decreasing and the exponential decreasing rate is regarded as an information quantity. Hence, even when $\mathcal{M}$ is general, it is expected that its asymptotic behavior gives an information quantity in the GPT with the measurement class $\mathcal{M}$. It would be an interesting problem whether such information quantities that characterize measurement classes.

Y. Y. was supported by Japan Society for the Promotion of Science (JSPS) Grant-in-Aid for JSPS Fellows No. 19J20161. M.H. was supported in part by Guangdong Provincial Key Laboratory (Grant No. 2019B121203002), JSPS Grant-in-Aids for Scientific Research (A) No. 17H01280 and for Scientific Research (B) No. 16KT0017, and Kayamori Foundation of Information Science Advancement.

*m17043e@math.nagoya-u.ac.jp

†m18003b@math.nagoya-u.ac.jp

thayashi@sustech.edu.cn

[1] P. Janotta and R. Lal, Phys. Rev. A 87, 052131 (2013).

[2] P. Janotta and H. Hinrichsen, J. Phys. A 47, 323001 (2014).

[3] Y. Yoshida and M. Hayashi, J. Phys. A 53, 215303 (2020).

[4] J. Bae, D. G. Kim, and L. Kwek, Entropy, 18, 39 (2016).

[5] K. Matsumoto and G. Kimura, arXiv:1802.01162.

[6] A. J. Short and S. Wehner, New J. Phys. 12, 033023 (2010).

[7] H. Barnum, J. Barrett, L. O. Clark, M. Leifer, R. Spekkens, N. Stepanik, A. Wilce, and R. Wilke, New J. Phys. 12, 033024 (2010).

[8] O. C. O. Dahlsten, D. Lercher, and R. Renner, New J. Phys. 14, 063024 (2012).

[9] M. P. Müller and C. Ududec, Phys. Rev. Lett. 108, 130401 (2012).

[10] M. P. Müller and L. Masanes, New J. Phys. 15, 053040 (2013).

[11] L. Masanes and M. P. Müller, New J. Phys. 13, 063001 (2011).

[12] J. G. Richens, J. H. Selby, and S. W. Al-Safi, Phys. Rev. Lett. 119, 080503 (2017).

[13] L. Lami, C. Palazuelos, and A. Winter, Commun. Math. Phys. 361, 661 (2018).

[14] G. Aubrun, L. Lami, C. Palazuelos, S. J. Szarek, and A. Winter, Commun. Math. Phys. 375, 679 (2020).

[15] C. M. Lee and J. Barrett, New J. Phys. 17, 083001 (2015).

[16] S. Popescu and D. Rohrlich, Found. Phys. 24, 379 (1994).

[17] M. Plávala and M. Ziman, Phys. Lett. A 384, 126323 (2020).

[18] H. Arai, Y. Yoshida, and M. Hayashi, J. Phys. A 52, 465304 (2019).

[19] See the Supplemental Material at http://link.aps.org/ supplemental/10.1103/PhysRevLett.125.150402 for full statements and their proofs. 